Ciencia, Ambiente y Clima, Vol. 4, No. 1, enero-junio, 2021 • ISSN (impreso): 2636-2317• ISSN (en línea): 2636-2333

DOI: https://doi.org/10.22206/cac.2021.v4i1.pp31-32

\title{
NOTA DEL II CICLO DE CONFERENCIAS EN CIENCIAS BIOLÓGICAS (II CCB), UNIVERSIDAD AUTÓNOMA DE SANTO DOMINGO, REPÚBLICA DOMINICANA, 2021
}

\section{Note of the II Cycle of Conferences in Biological Sciences (II CCB), Universidad Autónoma de Santo Domingo, Dominican Republic, 2021}

\section{Cristopher Jiménez-Orozco}

Instituto de Investigaciones Botánicas y Zoológicas Prof. Rafael M. Moscoso (IIBZ), Universidad Autónoma de Santo Domingo (UASD), República Dominicana. ORCID: 0000-0003-0343-6929

Correo-e: cristopheruasd@gmail.com

\section{J. A. Rojas}

Universidad Autónoma de Santo Domingo (UASD), República Dominicana. ORCID: 0000-0001-7232-5902

Correo-e: joel0.3@hotmail.com

\section{Recibido: 7/5/2021 • Aprobado: 25/6/2021}

Cómo citar: Jiménez-Orozco, C., \& Rojas, J. A. (2021). Nota del II Ciclo de Conferencias en Ciencias Biológicas (II CCB), Universidad Autónoma de Santo Domingo, República Dominicana, 2021. Ciencia, Ambiente y Clima, 4(1), 31-32. DOI: https://doi.org/10.22206/ cac.2021.v4i1.pp31-32

La segunda edición del Ciclo de Conferencias en Ciencias Biológicas (II CCB) tuvo lugar los días 26, 27 y 28 de mayo del año 2021. El evento se llevó a cabo de manera virtual a través de la plataforma Zoom, en el marco de las actividades del quincuagésimo quinto aniversario (55\%) de la Facultad de Ciencias de la Universidad Autónoma de Santo Domingo (UASD), con auspicios de la Asociación Dominicana de Estudiantes de Biología (ADEBIO), el Grupo de discusión científica Ekmanianthe, la Escuela de Biología y la Oficina Sectorial de Planificación y Desarrollo Institucional (OSEPLANDI) de la UASD.

El II CCB tuvo como objetivo promover la difusión de investigaciones y el acercamiento entre estudiantes y profesionales de las ciencias biológicas de la región. En esta segunda edición, el CCB fue dedicado a tres destacadas biólogas dominicanas: Jackeline Salazar Lorenzo, Ph. D. (botánica y bióloga sistemática), Jeannette Mateo Pérez, M. Sc. (oceanógrafa y bióloga pesquera) y Ruth $\mathrm{H}$. Bastardo Landrau, M. Sc. (entomóloga y ecóloga).

"Conectando la biodiversidad en las Américas: divulgación académica, educación e investigación" es el eslogan escogido para este II CCB, evidenciando la crítica necesidad de cooperación entre investigadores de la comunidad científica nacional e internacional en aras de la actual crisis por la pandemia del COVID-19, cuya sinergia resulta crucial para el desarrollo de las Instituciones de Educación Superior (IES) de República Dominicana, en términos de ciencia y tecnología.

El evento contó con la participación de 25 expositores (siete conferencistas magistrales), nacionales e internacionales, y aproximadamente 70 participantes, entre estos: estudiantes, docentes, investigadores y público en general. Dada la naturaleza 
multidisciplinaria del II CCB, se presentaron investigaciones correspondientes a las áreas de biología evolutiva y filogenética, biología de la conservación, biología molecular, briología, divulgación científica, epigenética, ecología urbana, herpetología, ictiología y pteridología.

Entre los ponentes magistrales se encuentra el ictiólogo dominicano Carlos Ml. Rodríguez Peña, Ph. D. [Ministerio de Educación Superior, Ciencia y Tecnología (MESCyT)], quien conferenció sobre los avances y desafíos actuales de los estudios filogenéticos en la tribu Poeciliini en la Hispaniola. Roman Ramos Báez (Department of Biology, University of Washington, Seattle), biólogo molecular dominicano, nos presentó una sinopsis de sus investigaciones sobre la evolución de receptores auxínicos en el maíz (Zea mays) utilizando levadura. En otro orden, la pteridóloga estadounidense Susan Fawcett, Ph. D. (University of California, Berkeley) nos condujo hacia una perspectiva integrativa de la taxonomía de pteridofitas, desentrańando las relaciones filogenéticas y los conceptos genéricos de la familia Thelypteridaceae; mientras que la brióloga panameña Noris Salazar Allen, Ph. D. (Smithsonian Institution) nos brindó un recuento de la taxonomía, los endófitos y la fitoquímica de las hepáticas neotropicales del género Cyathodium (Cyathodiaceae).

Por otro lado, el entomólogo español Pedro M. Alarcón Elbal, Ph. D. [Instituto de Medicina Tropical y Salud Global (UNIBE)], disertó sobre sus investigaciones en torno a la ocurrencia y vigilancia entomológica del vector Aedes vittatus Culicidae en las Américas, con énfasis en República Dominicana. Omar M. Entiauspe-Neto (Universidade Federal do Rio Grande, Brasil), herpetólogo brasileño, aportó una reseña de la historia natural y taxonomía de los anfibios y reptiles en el Neotrópico. Finalmente,
Luis M. Díaz Beltrán, Ph. D., herpetólogo cubano (Museo Nacional de Historia Natural, Cuba), presentó una panorámica del estado actual del conocimiento de los ofidios de Cuba.

Otras presentaciones se focalizaron en tópicos contemporáneos de biología de la conservación y cambio climático en las Antillas Mayores; este es el caso de Yommi Piña (biólogo dominicano), quien expone las problemáticas que amenazan los recursos florísticos de la Sierra de Bahoruco, al suroeste de República Dominicana, y Amelia Merced, Ph. D. (brióloga puertorriqueña), con un abordaje acerca de la situación actual de los estudios de las briofitas en Puerto Rico, particularmente sobre cómo las especies de Sphagnum (Sphagnaceae) pueden ser utilizadas como indicadores del cambio climático.

En el marco del II CCB se impartió exitosamente el seminario "Lizards in an Evolutionary Tree: Ecology and Adaptive Radiation of Anolis Lizards", a cargo del ilustre ecólogo evolutivo y herpetólogo estadounidense Jonathan Losos, Ph. D. (Washington University). También se realizó el panel "Divulgación Científica de las Ciencias Biológicas en República Dominicana", estelarizado por investigadores emergentes de la Escuela de Biología de la UASD y el Museo Nacional de Historia Natural de Santo Domingo (MNHNSD).

El CCB se ha ido concretizando y ha adquirido relevancia dentro de la comunidad nacional de biólogos, propiciando una vez más un escenario de colaboración y conectividad académica con otros investigadores de las distintas instituciones de educación superior del país y la región. En eventos futuros esperamos contar con el apoyo y la acreditación de diferentes organismos de ciencia y tecnología de la UASD y otras instituciones gubernamentales de República Dominicana. 